RESEARCH ARTICLE

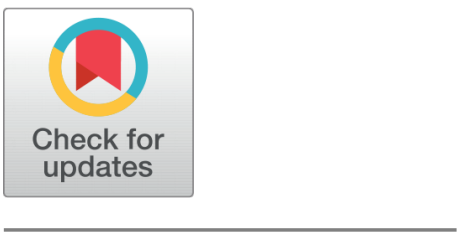

OPEN ACCESS

Received: 01.02.2021

Accepted: 10.03.2021

Published: 19.03.2021

Citation: Hosen F, Asif AKMAH, Hasan MZ, Mahmud ST, Islam MR (2021) Evaluation of comfort and thermal properties of stretch denim fabric by applying different softeners. Indian Journal of Science and Technology 14(8): 752-764. http s://doi.org/10.17485/IJST/v14i8.190

* Corresponding author.

ayatasif.mbstu@gmail.com

Funding: None

Competing Interests: None

Copyright: (c) 2021 Hosen et al. This is an open access article distributed under the terms of the Creative Commons Attribution License, which permits unrestricted use, distribution, and reproduction in any medium, provided the original author and source are credited.

Published By Indian Society for Education and Environment (iSee)

ISSN

Print: 0974-6846

Electronic: 0974-5645

\section{Evaluation of comfort and thermal properties of stretch denim fabric by applying different softeners}

\author{
Faruq Hosen ${ }^{1}$, A K M Ayatullah Hosne Asif ${ }^{*}$, Md. Zayedul Hasan ${ }^{1}$, \\ Sharif Tasnim Mahmud' ${ }^{1}$ Md. Rafiul Islam ${ }^{1}$ \\ 1 Department of Textile Engineering, Mawlana Bhashani Science and Technology University, \\ Santosh, Tangail, Bangladesh
}

\section{Abstract}

Denim plays a vital role in the fashion industry. But today's fashion not only concern about its aesthetic property but also its comfortability. Objective: This study was aimed to explore the effect of different softeners on the comfort and thermal feeling properties of stretch denim fabric. Method/analysis: For the understanding of comfort, feeling, and thermal properties of stretch denim fabric in conjunction with enzyme wash and stone enzyme wash; different softeners like cationic, anionic, nonionic, micro silicone, macro silicone, and nano-silicone softeners were applied accordingly. Then water vapor permeability and comfort property tests were conducted to understand the effect of different softeners on comfort and thermal properties of stretch denim fabric. Findings: Comfort, feeling, and thermal properties of stretch denim fabric were affected by using different kinds of softeners. Enzyme and stone enzyme washed stretch denim fabric treated with silicone-based softeners illustrated better feeling performance regarding the smoothness, softness, and warmness sensation. For water vapor permeability analysis, nonionic softeners showed better performance for both cases of enzyme wash and stone enzyme wash rather than others. Furthermore, nonionic and anionic softeners displayed lower performance for thermal conductivity during compression and recovery than silicone-based and cationic softeners in context with both Enzyme wash along with stone enzyme wash treated stretch denim fabric. Novelty: This research is a novel work regarding the understanding of comfort and thermal feeling properties of stretch denim fabric by applying different softeners together with enzyme wash and stone enzyme wash.

Keywords: Denim; softener; water vapor permeability; thermal conductivity; smoothness; warmness 


\section{Introduction}

It is needless to say that without denim; fashion is incomplete. No other fabric has been as widely accepted as denim in the history of textiles and it is the material of generations, used by people of all classes and ages ${ }^{(1,2)}$. Denim has undisputed position in the fashion industry and her ability to adapt creativeness in fashion trends through her uniqueness and technologically advanced washing effects. Various scientific and technological advancements have been utilized for over the years in the garments industry, which has initiated durability, together with comfortness, have made denim jeans extremely popular ${ }^{(3)}$. Technically denim is a durable cotton fabric, traditionally woven with indigo dyed warp and white fillings. Its manufacturing process involves the same classical principles that have been followed since its creation, but technological advancement have turned it into a modern fashion material ${ }^{(4)}$.

Denim is the oldest fashion products in the world and most often associated with jeans ${ }^{(5)}$. Stretch denim is a style of jeans also a new type of fabric mostly cotton-polyester blend which integrates a small amount of elastane known as spandex. Stretch denim usually constructed with indigo dyed warp and white weft thread alongside contains about $1-3 \%$ elastane ${ }^{(6)}$. The white threads extend along the entire width of the fabric. Currently, enzymes are mainly used to obtain a cleaner surface ${ }^{(7)}$. They have played a key role in providing an aesthetic finish and improving the attractiveness, reducing pills, increasing softness, smoothness, lusture, improving fabric maneuverability and coating ability, and improving vintage effects that meet current trend requirements in fashion-oriented jeans ${ }^{(8)}$. Softeners also play an important role regarding their main impact on the fabric surface. Furthermore, small portion of the softener molecules penetrate into the fiber and provide inner plasticization in order to reduce the glass transition temperature for the fiber-forming polymer ${ }^{(9)}$. Softening process in classical garments and denim washing are considered to be the preceding step before the drying process as softeners are widely used in finishing operations to improve fabric handling and other valuable properties. Softeners also create the exterior layer of the fabric with electrically charged chemical compounds providing threads to stand up from the surface ${ }^{(10,11)}$. It generates smoother and more fluffy feel to the fabric ${ }^{(12)}$.

Washing process in denim garments is considered as one of the essential parameters. This process augments a lot of value to the final product. There are several methods in the washing process to induce fading, vintage effects ${ }^{(13)}$. Washing is traditionally done by such kind of way or combinations involves enzyme washing, stone enzyme washing with strong bleaching agents such as sodium hypochlorite, potassium permanganate ${ }^{(14)}$. It is a harsh reality that several processes associated in textile manufacuring are not environmentally friendly ${ }^{(15)}$. Over the past few years, technological advancement took place regarding the sustainable washing, green dyeing for textile materials processing ${ }^{(16)}$. Moreover substitute approaches have also been developed to utilize cellulase enzymes instead of pumice stones to achieve an abrasive effect ${ }^{(17)}$. The newly developed denim of various designs enriched with softness, lightness and comfortable attributes have added a new dimension to the world of fashion in all seasons ${ }^{(18,19)}$.

Comfort properties in clothing are considered as one of the essential quality criteria for consumers. Therefore, it is necessary to take into account the features of comfort in textiles ${ }^{(20,21)}$. Ease of wearing; an element that contains many factors, such as aesthetic comfort, thermal comfort, ease of exercise and psychological flexibility. Thermal comfort is defined as a feeling of enjoyment man feels in his environment. It depends on heat and mass transfer between the body and the environment associated with thermal resistance and water vapor permeability clothing ${ }^{(22)}$. Features of modern clothing provide comfort and protect clothing flexibility in different environmental conditions. The Insulation properties in the garment must also have water vapor permeability at any level and to uphold body temperature to maintain comfort; otherwise, a feeling of anxiety arises ${ }^{(23,24)}$. Given the characteristics of clothing such as heat resistance, water vapor permeability, and feeling performance plays a significant role in product development ${ }^{(25)}$. When it's too hot during the production level in the human body; higher than usual, sweating and evaporation of sweat responsible for the surface of the skin lose heat ${ }^{(26)}$. Liquid sweat accumulates on the surface of the skin or on the inner layer of the garment to be transferred an external environment that allows the liquid to evaporate and to prevent the sensation of moisture ${ }^{(27,28)}$. In the clothing system, when concentration of water evaporation exceeds the saturation level, all condensation occurs for local heating throughout the layers of the clothing system causes a disturbing sensation ${ }^{(29,30)}$. Fundamentally, the sweat can be evaporated during the cooling; thus high level of moisture transmission and evaporation should always be programmed by clothing ${ }^{(31)}$. The principal objective of this investigation was to discover the comfort, thermal, feeling properties of the stretch denim fabric treated with enzyme wash and stone enzyme washing in context with the effect of different softeners. 


\section{Materials and Methods}

\subsection{Materials}

\subsubsection{Fabric}

For experimentation, $98 \%$ of cotton $2 \%$ of elastane indigo-dyed stretch denim fabric was used. The fabric GSM (grams per square meter) was 330 (Before wash), 3/1 right-hand twill fabric with width 57 inch.

\subsubsection{Chemicals}

Desizing agent sodium carbonate was used in this experiment. This chemical was imported from China. Detergent (Suntex, HF) was imported from Germany. Enzyme (HIDROS TED, neutral enzyme-liquid form), different softeners like cationic, anionic, nonionic, silicone (micro, macro, nano) softeners were also used for this study.

\subsubsection{Experimental instruments}

To perform this experiment, washing machine, hydro extractor machine, tumble dryer machine, fabric GSM cutter, electronic balance and fabric $\mathrm{pH}$ meter was used.

\subsection{Methodology}

\subsubsection{Working principle of enzyme wash}

Enzyme washing is such kind of laundering process utilizes enzyme to soften and finish fabric; providing jeans and other garments with a worn-in look and feel. Enzyme washing is ecologically friendly due to the natural origins of enzymes, which is acted like biodegradable. The chronological steps of operations involved in enzyme wash are listed below:

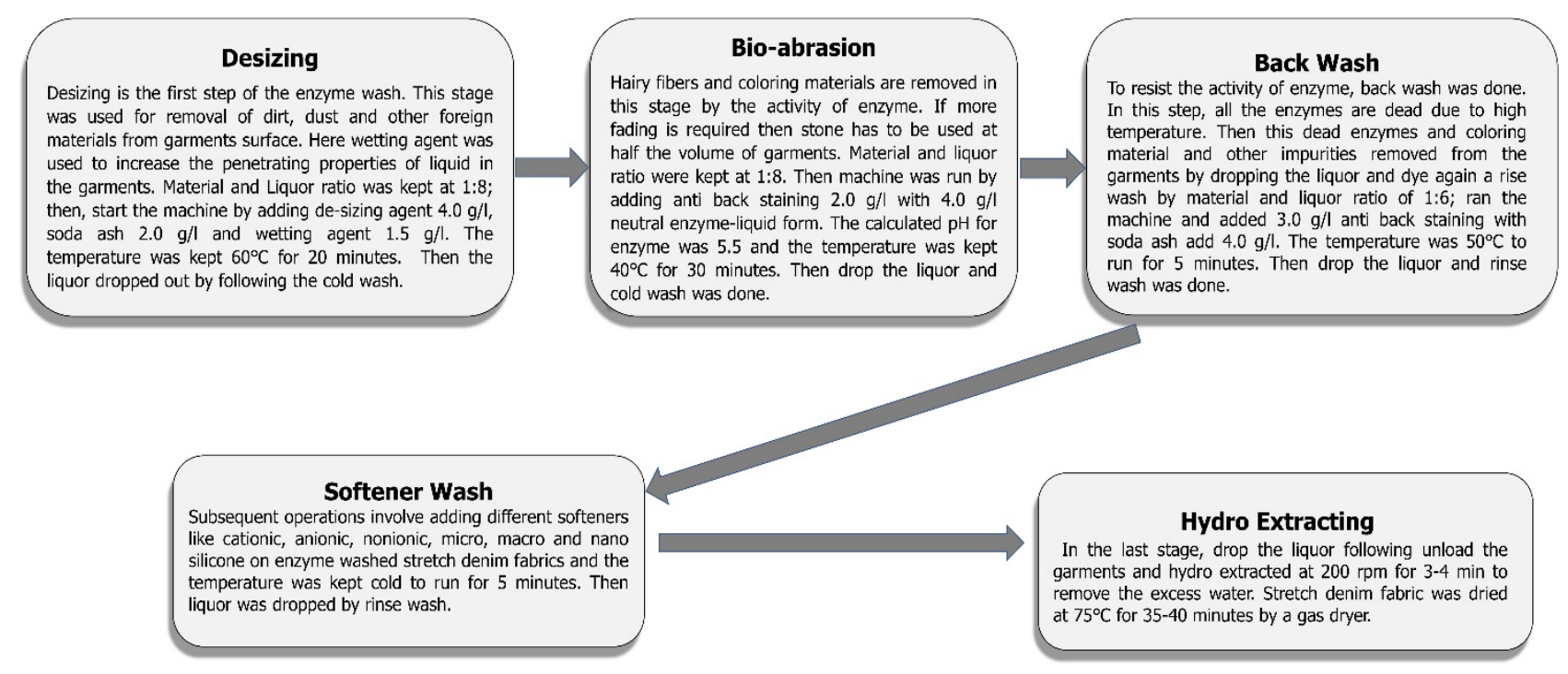

\subsubsection{Working principle of stone enzyme wash}

Stone washing process on stretch denim fabric have been used to produce faded, worn-out appearance. This stone washing process was carried through washing the stretch denim fabric with pumice stones in a rotating drum by using chemicals to create the necessary outlook. For this experimentation, stone washing process was conducted by desizing, bio-abrasion, addition of pumice stones with enzyme, acetic acid and antistain at $40-50^{\circ} \mathrm{C}$ for $40-60$ mins followed by tumbling for $40-50$ mins, drain the liquor followed by separation of garments from stones, softening and hydro extracting procedures.

\subsubsection{Determination of water vapor permeability}

The water vapor permeability test was determined according to British Standards (BS 3424-34) ${ }^{(32)}$.

Testing Method: Cup Method 
Testing Conditions: Temperature $=(20 \pm 2)^{\circ} \mathrm{C}$, Relative humidity $=(65 \pm 2) \%$

Sample size: $175 \mathrm{~mm} \times 100 \mathrm{~mm}$

Apparatus: Aluminum cup, beaker, incubator, electronic balance

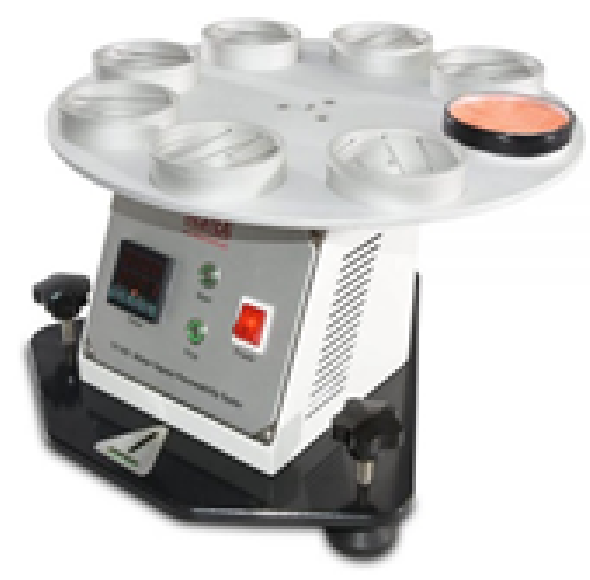

Fig 1. Water vapor permeability testing machine

\subsubsection{Determination of fabric comfort properties}

Fabric comfort properties were determined according to International Organization for Standardization (ISO 11092: 2014) $\operatorname{method}^{(33)}$.

Test Specimen Size: $310 \mathrm{~mm} \times 310 \mathrm{~mm}$ letter "L" width $=110 \mathrm{~mm}$; cross area $=110 \mathrm{~mm} \times 110 \mathrm{~mm}$

Testing Conditions: Temperature $=(20 \pm 2)^{\circ} \mathrm{C}$, Relative humidity $=(65 \pm 2) \%$

Apparatus: Fabric touch tester (SDLATLAS), electronic balance

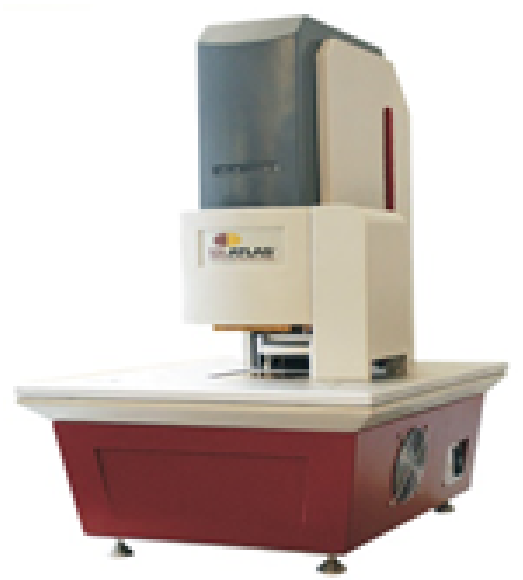

Fig 2. Fabric touch tester machine for testing comfort properties 


\section{Results and Discussion}

\subsection{Effect of different softeners on water vapor permeability of enzyme treated stretch denim fabric}

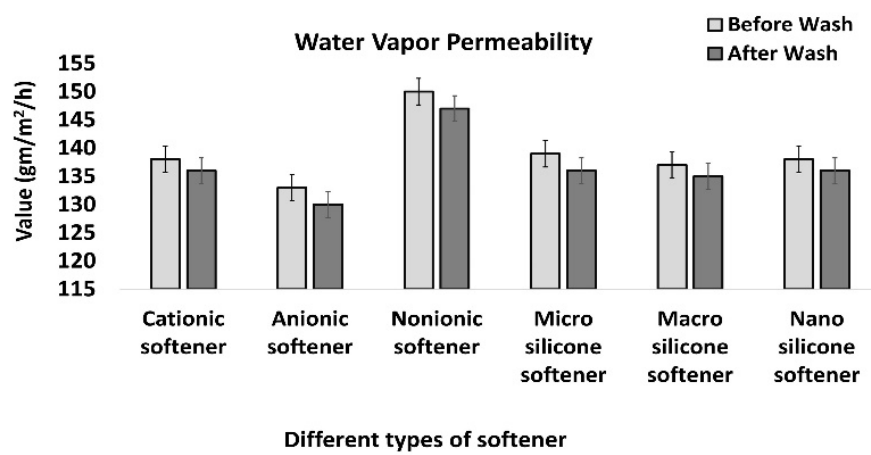

Fig 3. Variation of softeners on water vapor permeability of enzyme treated stretch denim fabric

The above figure represents about different softeners effect on water vapor permeability of enzyme treated stretch denim fabric. It was observed that the water vapor permeability was relatively better for enzyme wash treated stretch denim fabric with nonionic softener. The reason behind that, nonionic softeners showed superiority on hydrophilicity. Cationic and anionic softeners displayed lower value because these softeners orient themselves with the hydrophobic portion away from the fabric, thus offering lower performance for water vapor permeability test.

\subsection{Effect of different softeners on water vapor permeability of stone enzyme treated stretch denim fabric}

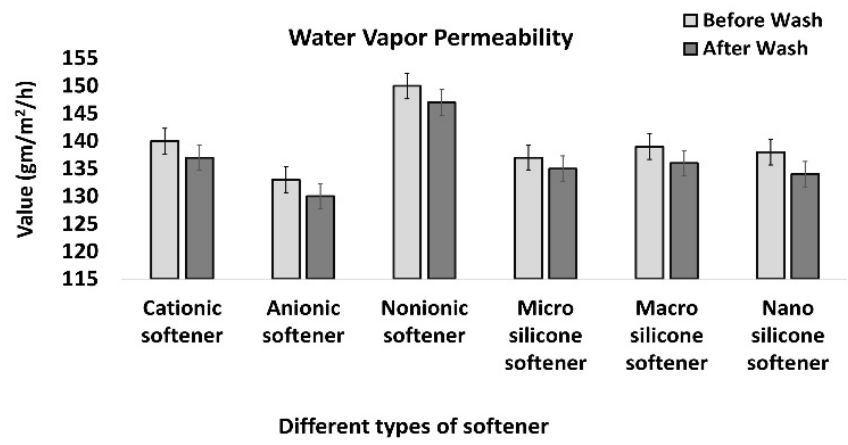

Fig 4. Variation of softeners on water vapor permeability of stone enzyme treated stretch denim fabric

The following figure demonstrates about the effect of different softeners on water vapor permeability of stone enzyme treated stretch denim fabric. It was observed that the water vapor permeability was relatively better for stone enzyme wash treated stretch denim fabric with nonionic softener. The reason behind that nonionic softeners shows superiority on hydrophilicity. In both enzyme wash and stone enzyme wash; cationic softeners and anionic softeners displayed lower value because these softeners orient themselves with the hydrophobic portion away from the fabric, thus offering lower performance for water vapor permeability test. 


\subsection{Effect of various softeners on thermal conductivity during compression of enzyme treated stretch denim fabric}

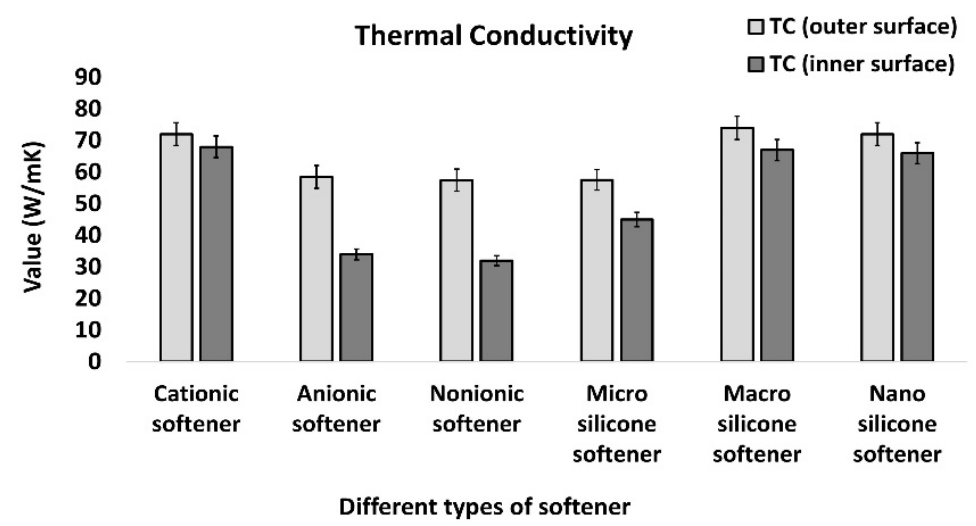

Fig 5. Variation of softeners on thermal conductivity during compression of enzyme treated stretch denim fabric

Above figure illustrates about the effect of softeners on thermal conductivity during compression of enzyme treated stretch denim fabric. It was detected that transmission of energy per meter when specimen was under compression showed the highest rate in enzyme wash with cationic and macro silicone softener treated stretch denim in inner surface but lowest in enzyme wash with anionic, nonionic softener and micro silicone softener in inner surface. Macro silicone softener specifically provides an exceptional, unique hand, high lubricity and cationic softener represents durable to multiple washes and imparts antistatic properties.

\subsection{Effect of various softeners on thermal conductivity during compression of stone enzyme treated stretch denim fabric}

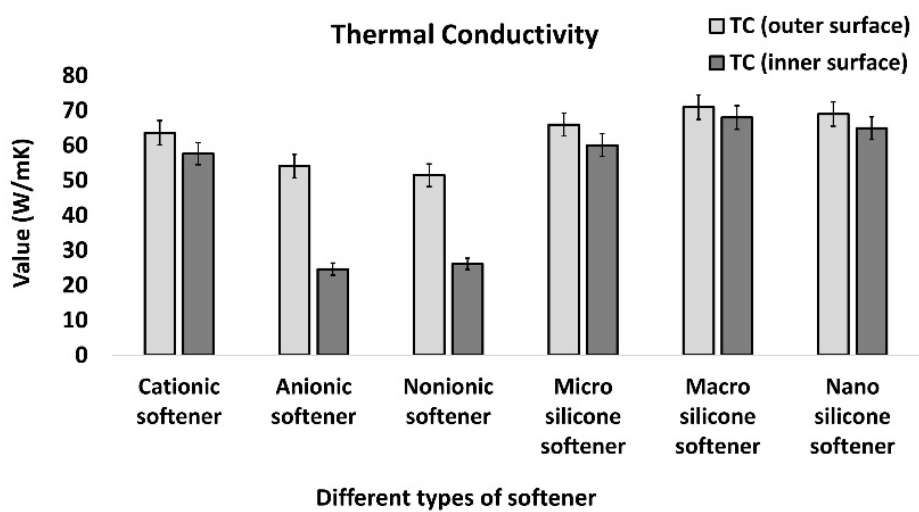

Fig 6. Variation of softeners on thermal conductivity during compression of stone enzyme treated stretch denim fabric

The following figure demonstrates the effect of various softeners on thermal conductivity during compression of enzyme stone treated stretch denim fabric. It was observed that transmission of energy per meter when specimen was under compression showed the highest rate in enzyme stone wash with macro silicone softener treated stretch denim in outer and inner surface but lowest in enzyme stone wash with anionic, nonionic softeners in inner surface. In both cases of enzyme wash and stone enzyme wash, cationic and silicone-based softeners represented better performance than others due to quickly let the heat pass from a hot side to a cooler side. 


\subsection{Effect of softeners on thermal conductivity during recovery of enzyme treated stretch denim fabric}

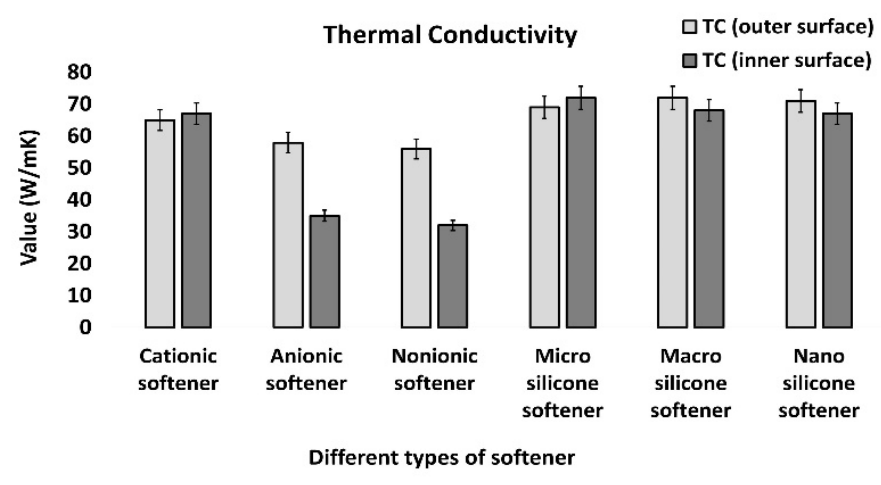

Fig 7. Variation of softeners on thermal conductivity during recovery of enzyme treated stretch denim fabric

Above figure illustrates about the effect of different softeners on thermal conductivity during recovery of enzyme treated stretch denim fabric. It was observed that transmission of energy per meter when the specimen recovered showed the highest value in case of enzyme wash with micro silicone softener treated stretch denim in inner and outer surface. In contrast, the lowest value was observed in enzyme wash with anionic and nonionic softener treated stretch denim in inner and outer shell.

\subsection{Effect of softeners on thermal conductivity during recovery of stone enzyme treated stretch denim fabric}

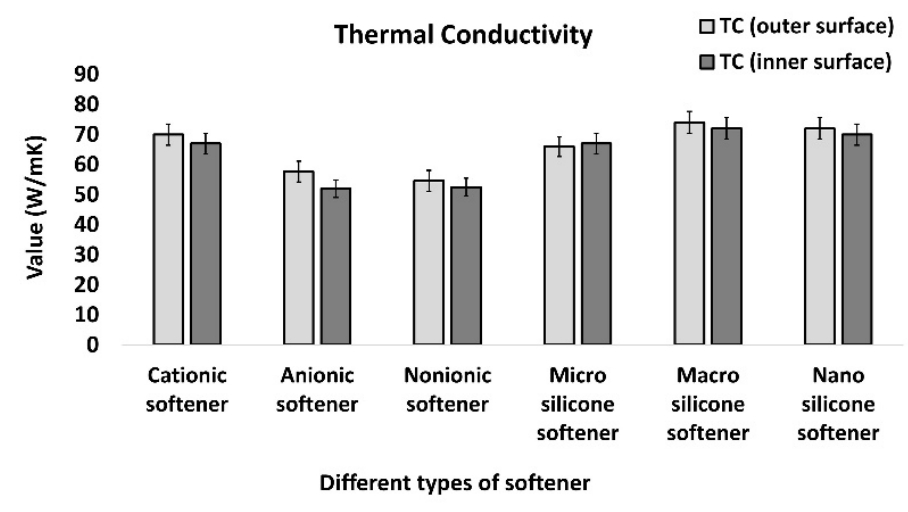

Fig 8. Variation of softeners on thermal conductivity during recovery of stone enzyme treated stretch denim fabric

The following figure demonstrates about different softeners consequence on thermal conductivity during the recovery of stone enzyme treated stretch denim fabric. It was observed that transmission of energy per meter when the specimen recovered represented the highest value in case of enzyme stone wash with macro silicone softener treated stretch denim in the outer and inner surface. In contrast, the lowest value was observed in stone enzyme wash with anionic and nonionic softener treated stretch denim in the inner and outer surface. Macro silicone softener provided exceptional, unique hand, high lubricity and high degree of permanence that form cross-linked films and a range of properties from hydrophobic to hydrophilic. Anionic and nonionic softeners epitomized lower values than silicone-based and cationic softeners in context with both enzyme wash along with stone enzyme wash treated stretch denim fabric. In both cases of enzyme wash and stone enzyme wash, cationic and silicone-based softeners represent better performance than others due to quickly let the heat pass from a hot side to a cooler side (the air on the other side of the denim). 


\subsection{Effect of different softeners on thermal flux of enzyme treated stretch denim fabric}

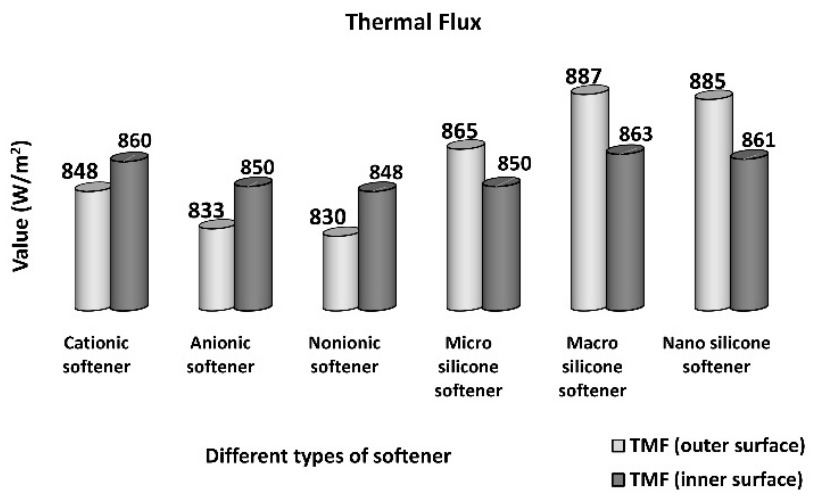

Fig 9. Variation of softeners on the thermal flux of enzyme treated stretch denim fabric

The above figure represents about the effect of different softeners on the thermal flux of enzyme treated stretch denim fabric. Maximum energy transmitted in stretch denim fabric was accompanied by enzyme wash with macro and nano silicone softeners in outer and inner surface. By using silicone-based softeners, stretch denim fabric can achieve pliable, soft hand and smoothness attributes.

\subsection{Effect of different softeners on thermal flux of stone enzyme treated stretch denim fabric}

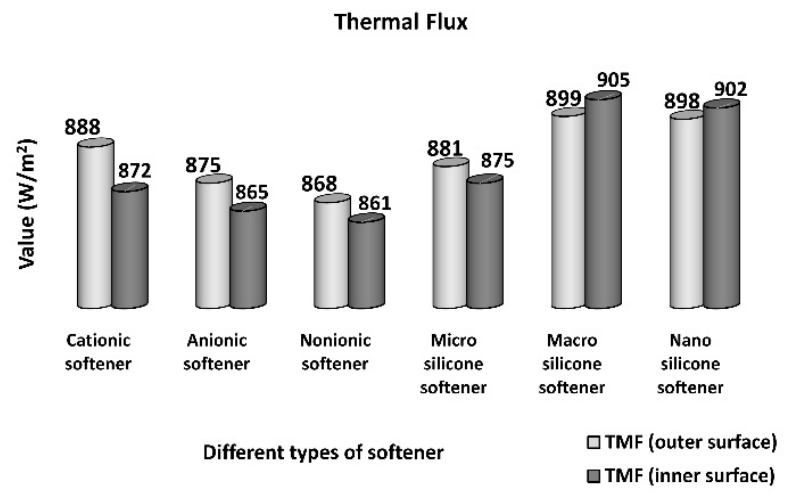

Fig 10. Variation of softeners on thermal flux of stone enzyme treated stretch denim fabric

The following figure demonstrates about the effect of softeners on thermal flux of enzyme stone treated stretch denim fabric. Maximum energy transmitted in stretch denim fabric was accompanied by stone enzyme wash with macro silicone softener in outer and inner surface. In both enzyme wash and stone enzyme wash, silicone-based softeners and cationic softener represent better performance than others because by using silicone-based softeners; fabrics can achieve an agreeable, soft hand which enhanced the smoothness, flexibility, drape and pliability of the stretch denim fabric greatly. The cationic softener also displayed acceptable results. 


\subsection{Effect of various softeners on smoothness performance of enzyme treated stretch denim fabric}

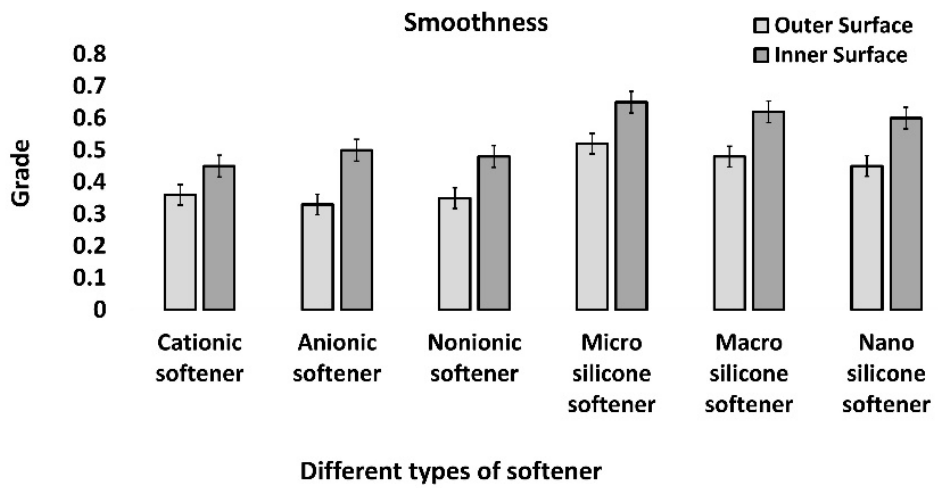

Fig 11. Variation of softeners on the smoothness of enzyme treated stretch denim fabric

Above figure represents about the effect of various softeners on the smoothness of enzyme treated stretch denim fabric. Smoothness is the touch sensation which indicates how even and regular surface the fabrics have. Fabric touch tester provided a numerical value which represented that, enzyme wash with silicone-based softeners specifically micro silicone treated stretch denim in outer surface and inner surface had the highest smoothness rating rather than others due to creating a lubricating and moderately waterproof film on the surface and furnished fabric a silky, fluffy hand.

\subsection{Effect of various softeners on smoothness performance of stone enzyme treated stretch denim fabric}

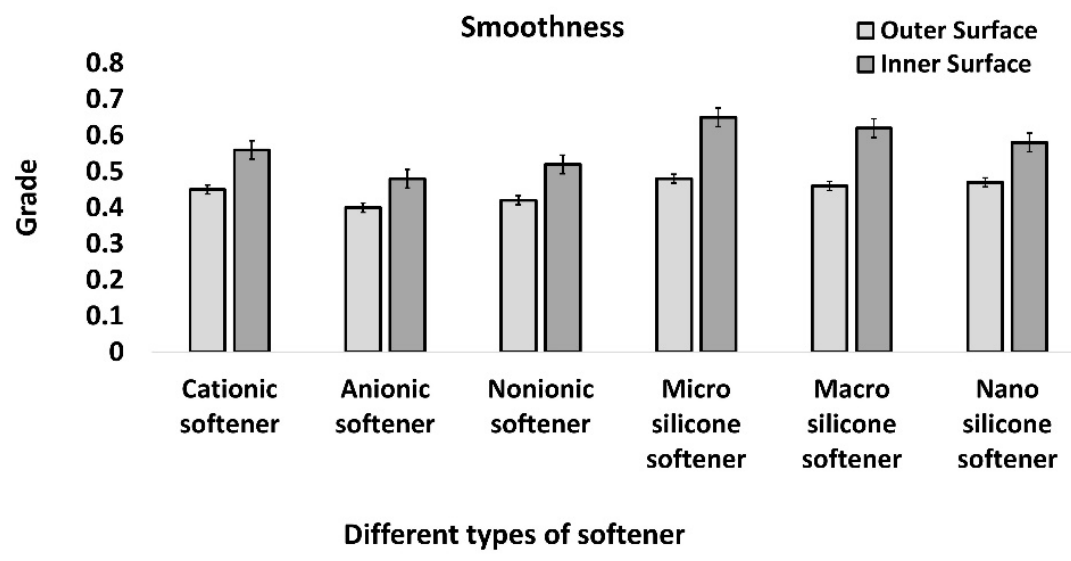

Fig 12. Variation of softeners on the smoothness of stone enzyme treated stretch denim fabric

The following figure epitomizes about the effect of different softeners on the smoothness of stone enzyme treated stretch denim fabric. Enzyme stone wash with micro and nano silicone softeners treated stretch denim in the outer surface, and the inner surface had the highest smoothness rather than other softeners treated. In both cases of enzyme wash and stone enzyme wash, silicone-based softeners represented better performance than others due to creating a lubricating and moderately 
waterproof film on the surface and furnished the fabric like a soft, silky, fluffy hand.

\subsection{Effect of different softeners on warmness of enzyme treated stretch denim fabric}

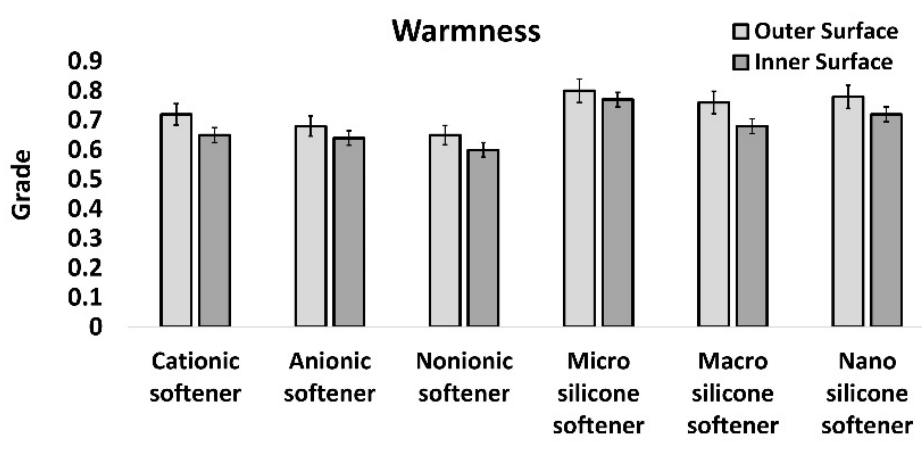

Different types of softener

Fig 13. Variation of softeners on warmness of enzyme treated stretch denim fabric

The following figure demonstrates about the effect of different softeners on warmness sensation of enzyme treated stretch denim fabric. From the following values, it can be said that enzyme with silicone-based softeners treated stretch denim fabric showed more warmness grade than cationic, anionic, nonionic softeners. Anionic and nonionic softeners provided lower performance than cationic softener due to their less durability and less softness performance. On the other hand, siliconebased softeners demonstrated better results than others because of good temperature stability and durability, with a high degree of permanence that form cross-linked films and a range of properties from hydrophobic to hydrophilic.

\subsection{Effect of different softeners on warmness of stone enzyme treated stretch denim fabric}

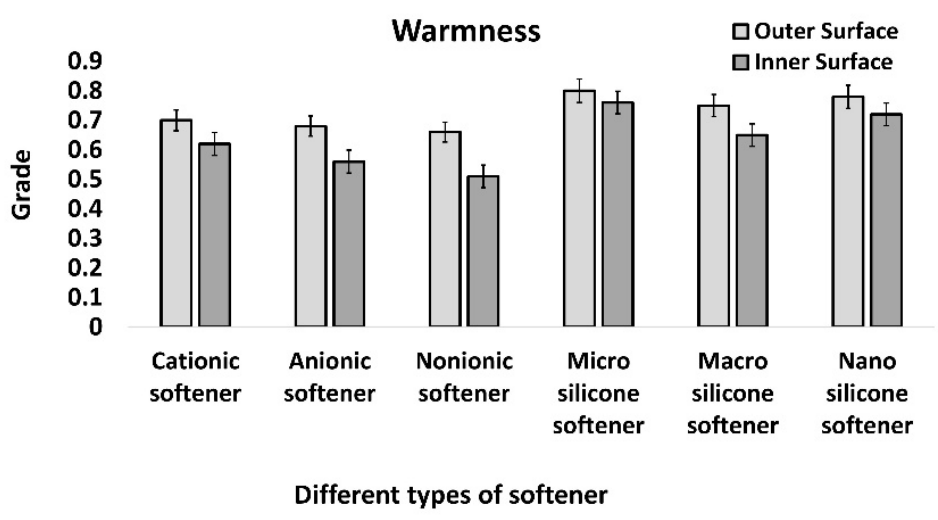

Fig 14. Variation of softeners on warmness of stone enzyme treated stretch denim fabric

The above figure determines about the effect of different softeners on warmness sensation of stone enzyme treated stretch denim fabric. Stone enzyme with silicone-based softeners treated stretch denim fabric showed more warmness grade than cationic, anionic, nonionic softeners. Anionic and nonionic softeners displayed lower grade performance in both cases of enzyme wash and stone enzyme wash than cationic and silicone-based softeners due to their less durability and less softness 
performance. On the other hand, in both cases of enzyme wash and stone enzyme wash; silicone-based softeners demonstrated better performance rather than others because of good temperature stability and durability, with high degree of permanence that form cross linked films and a range of properties from hydrophobic to hydrophilic.

\subsection{Effect of different softeners on softness performance of enzyme treated stretch denim fabric}

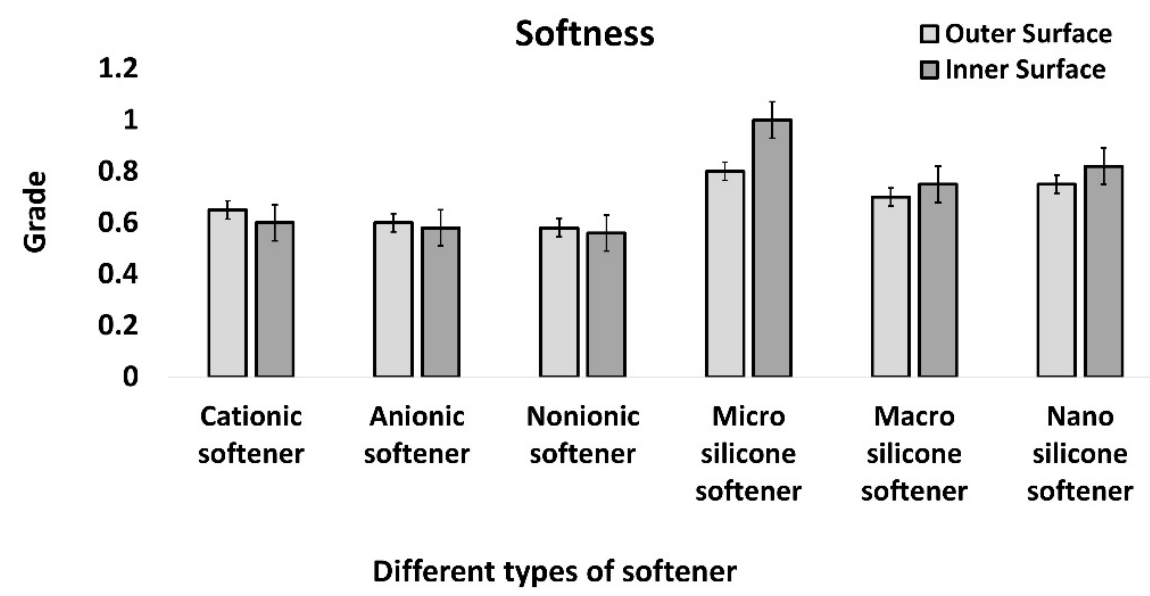

Fig 15. Variation of softeners on the softness performance of enzyme treated stretch denim fabric

The following figure demonstrates about the effect of softeners on feeling property like softness performance of enzyme treated stretch denim fabric. Softness indicates how much a fabric is easy to mold, fold or compress, not hard or firm to touch. From the following values, for stretch denim fabric treated with enzyme wash with micro and nano silicone softeners represented better softness performance (inner surface and outer surface) than the other softeners.

\subsection{Effect of softeners on softness performance of stone enzyme treated stretch denim fabric}

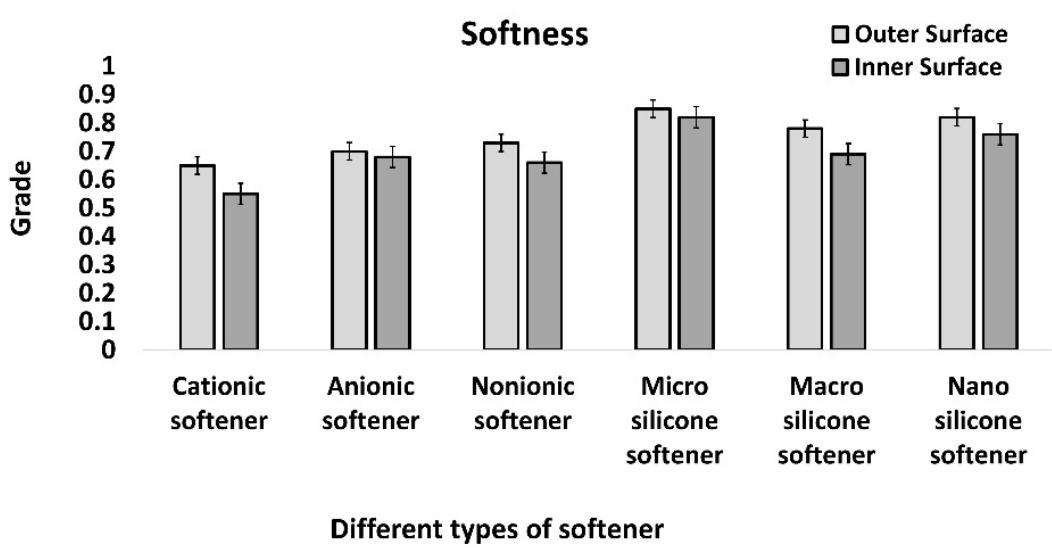

Fig 16. Variation of softeners on the softness performance of stone enzyme treated stretch denim fabric 
The above figure indicates about the effect of softeners on feeling property like softness performance of enzyme treated stretch denim fabric. Enzyme wash with micro and nano silicone softeners treated stretch denim fabric represented better softness performance (inner surface and outer surface) than the other softeners treated stretch denim fabric. Moreover, in both cases of enzyme wash and stone enzyme wash; silicone-based softeners demonstrated better performance rather than others because silicone-based softeners imparted unique soft handle with supple, pliable and fluffy effect on fabric surface rather than other softeners.

\section{Conclusion}

In this research work, different commercially available softeners like cationic, anionic, nonionic, micro, macro and nano silicone softeners were applied on stretch denim fabrics for enzyme wash and stone enzyme wash condition to investigate the comfortability, thermal and feeling properties. Specifically, stretch denim was selected for this experimentation; because this type of denim is considered as one of the most popular types of denim fabrics right now in the modern fashion world. The enzyme and stone enzyme washed sample treated with silicone-based softeners displayed better performance regarding the feeling performance. For water vapor permeability analysis, nonionic softeners demonstrated better performance rather than cationic, anionic, silicone-based softeners. In both cases of enzyme wash and stone enzyme wash; cationic softeners and anionic softeners displayed lower values for water vapor permeability. Anionic and nonionic softeners showed lower values for thermal conductivity during compression and recovery in context with both enzyme wash along with stone enzyme wash treated stretch denim fabric. For overall perspectives like comfortability, thermal, feeling sensation; cationic and silicone-based softeners represented superior performance than other softeners like anionic and nonionic softeners. Based upon the findings, manufacturers and engineers will be more cautious about the use of appropriate softeners for the finishing treatment of stretch denim fabric. Such kind of understanding will help the end users and producers to achieve the best conceivable result from the finished goods based on comfort and thermal sensitivity.

\section{Compliance with ethics requirements}

This article does not contain any studies with human or animal subjects performed by any of the authors.

\section{Conflict of interest}

The authors have declared no conflict of interest.

\section{Acknowledgements}

The authors gratefully acknowledge the Department of Textile Engineering of Mawlana Bhashani Science and Technology University (MBSTU) and Dhaka University of Engineering and Technology (DUET) for technical support of this work.

\section{References}

1) Kumari A, Khurana K. Regenerated Cellulose-Based Denim Fabric for Tropical Regions: An Analytical Study on Making Denim Comfortable. Journal of Textiles. 2016;2016:1-10. Available from: https://dx.doi.org/10.1155/2016/4614168.

2) Du W, Zuo D, Gan H, Yi C. Comparative Study on the Effects of Laser Bleaching and Conventional Bleaching on the Physical Properties of Indigo Kapok/Cotton Denim Fabrics. Applied Sciences. 2019;9(21):4662-4662. Available from: https://dx.doi.org/10.3390/app9214662.

3) Asif AK, Hasan MZ. Application of nanotechnology in modern textiles: A review. International Journal of Current Engineering and Technology. 2018;8(2):227-231. Available from: https://doi.org/10.14741/ijcet/v.8.2.5.

4) Asif AKMAH. An Overview of Sustainability on Apparel Manufacturing Industry in Bangladesh. Science Journal of Energy Engineering. 2017;5(1). Available from: https://dx.doi.org/10.11648/j.sjee.20170501.11.

5) Lale M. Definition, history of denim fabric and Turkey's denim clothing exportin figures. Industria Textila. 2018;69:334-337. Available from: https://dx.doi.org/10.35530/it.069.04.1420.

6) Babaarslan O, Telli A. A Study on Usage of Chenille Yarn in Denim Fabric Production. 2013;20(92):1-10. Available from: https://doi.org/10.7216/ 130075992013209201.

7) Zeng B, Wang X, Byrne N. Development of cellulose based aerogel utilizing waste denim-A Morphology study. Carbohydrate Polymers. 2019;205:1-7. Available from: https://dx.doi.org/10.1016/j.carbpol.2018.09.070.

8) Talebi S, Montazer M. Denim Fabric with Flame retardant, hydrophilic and self-cleaning properties conferring by in-situ synthesis of silica nanoparticles. Cellulose. 2020;27(11):6643-6661. Available from: https://dx.doi.org/10.1007/s10570-020-03195-6.

9) Ibrahim NA, Eid BM, Aziz MS, Hamdy SM, Allah SEA. Green surface modification and nano-multifunctionalization of denim fabric. Cellulose. 2018;25(10):6207-6227. Available from: https://doi.org/10.1007/s10570-018-1985-y.

10) Eryuruk SH. The effects of elastane and finishing processes on the performance properties of denim fabrics. International Journal of Clothing Science and Technology. 2019;31(2):243-258. Available from: https://dx.doi.org/10.1108/ijcst-01-2018-0009. 
11) Choudhury AKR, Chatterjee B, Saha S, Shaw K. Comparison of performances of macro, micro and nano silicone softeners. Journal of the Textile Institute. 2012;103(9):1012-1023. Available from: https://dx.doi.org/10.1080/00405000.2012.654666.

12) Ertaş OG, Ünal BZ, Çelik N. Analyzing the effect of the elastane-containing dual-core weft yarn density on the denim fabric performance properties. The Journal of the Textile Institute. 2016;107(1):116-126.

13) Sabir E, Kadem FD. Comfort and Performance Properties of Raised and Laminated Denim Fabrics. Fibres and Textiles in Eastern Europe. 2016;24:88-94. Available from: https://dx.doi.org/10.5604/12303666.1198064.

14) Hosen F, Hasan MZ, Asif AA. Effect of Different Softeners on Dimensional Stability and Color Fastness Properties of Stretch Denim Fabric. Advances in Applied Sciences. 2020;5(4):112-119. Available from: http://doi.org/10.11648/j.aas.20200504.13.

15) Sarker P, Asif AKMAH, Rahman M, Islam MM, Rahman KH. Green Dyeing of Silk Fabric with Turmeric Powder Using Tamarind Seed Coat as Mordant. Journal of Materials Science and Chemical Engineering. 2020;08(02):65-80. Available from: https://dx.doi.org/10.4236/msce.2020.82007.

16) Eryuruk SH. Analyzing Thermophysiological Comfort and Moisture Management Behavior of Cotton Denim Fabrics. Autex Research Journal. 2020;0(0):1-7. Available from: https://dx.doi.org/10.2478/aut-2019-0073.

17) Rogale SF, c MB, Rogale D. Investigation of resistance to the passage of heat for different men's clothing combinations. In: Proceedings of the 10th Scientific-Professional Symposium Textile Science \& Economy. 2017.

18) Wang $\mathrm{L}, \mathrm{Lu} \mathrm{Y}, \mathrm{He}$ J. On the effectiveness of temperature-responsive protective fabric incorporated with shape memory alloy (SMA) under radiant heat exposure. Clothing and Textiles Research Journal. 2020;38(3):212-224.

19) Iqbal MS, Mamun A, Siddiquee MA, Asif AA. Effect of Finishing Machine Parameters on Dimensional Stability of Single Lacoste Cotton Knitted Fabric. Advances in Materials. 2016;5(5):35-43. Available from: https://doi.org/10.11648/j.am.20160505.12.

20) Özkan ET, Kaplangiray B. Investigating comfort properties of $3 / 1 \mathrm{Z}$ twill weaved denim fabrics. IOP Conference Series: Materials Science and Engineering. 2017;254(18). Available from: https://dx.doi.org/10.1088/1757-899x/254/18/182013.

21) Eryuruk SH. Effect of Fabric Layers on Thermal Comfort Properties of Multilayered Thermal Protective Fabrics. Autex Research Journal. 2019;19(3):271278. Available from: https://dx.doi.org/10.1515/aut-2018-0051.

22) Uren N, Okur A. Analysis and improvement of tactile comfort and low-stress mechanical properties of denim fabrics. Textile Research Journal. 2019;89(2324):4842-4857. Available from: https://dx.doi.org/10.1177/0040517519840634.

23) Değirmenci Z, Çelik N. Investigation of Thermal Comfort Properties of Jeggings Manufactured by the Use of Knitted Denim-Like Fabrics. Journal of Testing and Evaluation. 2016;44(1). Available from: https://dx.doi.org/10.1520/jte20140165.

24) Mangat MM, Hes L. Comfort aspects of denim garments. In: and others, editor. Denim. Woodhead Publishing. 2015;p. 461-479. Available from: https://doi.org/10.1016/b978-0-85709-843-6.00015-9.

25) Hasan SM, Asif AK, Mahmud ST, Shanewaz AK. An Experimental Overview of Seam Performance for Different Types of Denim Fabrics. International Journal of Current Engineering and Technology. 2020;10(5):740-748. Available from: https://doi.org/10.14741/ijcet/v.10.5.8.

26) Siddiquee MA, Asif AA, Khan RH, Anwar MT, Islam MS, Noushin N. Study on the Effect of Dyeing and Finishing Parameters on Cotton Knitted Two Thread Fleece Fabric and 1x1 Rib Fabric. Science Research. 2016;4(1):7-10. Available from: https://doi.org/10.11648/j.sr.20160401.12.

27) Rogale D, Majstorović G, Rogale SF. Comparative Analysis of the Thermal Insulation of Multi-Layer Thermal Inserts in a Protective Jacket. Materials. 2020;13(12). Available from: https://dx.doi.org/10.3390/ma13122672.

28) Rajwin AJ, Prakash C. Effect of Air Plasma Treatment on Thermal Comfort Properties of Woven Fabric. International Journal of Thermophysics. 2017;38(11). Available from: https://dx.doi.org/10.1007/s10765-017-2299-2.

29) Dhouib S, Khedher F, Sakli F. A new approach to predict the fabric shrinkage in denim garments after finishing treatments. The Journal of The Textile Institute. 2016;107:364-375. Available from: https://dx.doi.org/10.1080/00405000.2015.1034928.

30) Eryuruk SH. The effects of elastane and finishing properties on wicking, drying and water vapour permeability properties of denim fabrics. International Journal of Clothing Science and Technology. 2019;32(2):208-217. Available from: https://dx.doi.org/10.1108/ijcst-01-2019-0003.

31) Hasan MZ, Asif AKMAH, Razzaque A, Hasan MR, Sur S, Faruque MO. An Experimental Investigation of Different Washing Processes on Various Properties of Stretch Denim Fabric. Journal of Materials Science and Chemical Engineering. 2021;09(01):1-15. Available from: https://dx.doi.org/10.4236/ msce.2021.91001.

32) BS 3424-34: Testing Coated Fabrics - Part 34: Method 37. Method for Determination of Water Vapour Permeability Index (WVPI). .

33) ISO 11092, 2014. Textiles - Physiological effects - Measurement of thermal and water-vapour resistance under steady-state conditions (sweating guarded-hotplate test). . 